\title{
Gasto urinario alto como predictor de hipoparatiroidismo tras tiroidectomía total
}

\author{
High urinary output as a predictor of hypoparathyroidism post total-thyroidectomy \\ Daniel Garay-Lechuga1, Mónica León-González¹, Isabela Ramírez-Mulhern²* y Enrique R. Jean-Silver ${ }^{1}$ \\ ${ }^{1}$ Servicio de Cirugía General, American British Cowdray Medical Center, Ciudad de México; ${ }^{2}$ Escuela de Medicina y Ciencias de la Salud, \\ Tecnológico de Monterrey, Monterrey, Nuevo León. México
}

\section{Resumen}

Objetivo: Determinar la utilidad del gasto urinario alto como predictor temprano de bajo costo para hipoparatiroidismo posoperatorio. Método: Se realizó un estudio retrospectivo unicéntrico a 1 año en adultos sometidos a tiroidectomía total en The American British Cowdray Medical Center I.A.P., calculando el gasto urinario en 24 horas, utilizando valores de calcio sérico corregido por albúmina con una tabla de correlación e identificando a los pacientes que recibieron calcio suplementario en las primeras horas de posoperatorio. Se dividieron en pacientes con hipoparatiroidismo posoperatorio y con valores normales de calcio en el primer día, diferenciando a los que recibieron suplementación oral de calcio profiláctico. Resultados: Se estudiaron 47 pacientes, 19 (40\%) en el grupo con hipocalcemia posoperatoria y 28 (59.5\%) en el grupo sin hipocalcemia posoperatoria. La media de gasto urinario en las primeras 8 horas de posoperatorio fue mayor en el grupo de hipocalcemia posoperatoria en comparación con el grupo sin hipocalcemia, sin diferencia significativa $(p=0.392)$, y tampoco durante las primeras 16 horas $(p=0.435)$. Conclusiones: En nuestro estudio no existe relación entre el incremento del gasto urinario y la predicción de hipoparatiroidismo posoperatorio. Se necesitan estudios con muestras de mayor tamaño y con un diseño metodológico más fuerte (prospectivo) para determinar si en realidad la diferencia obtenida puede figurar como predictor.

Palabras clave: Hipocalcemia. Hipoparatiroidismo. Hormona paratiroidea. Tiroidectomía.

\section{Abstract}

Objective: to determine the usefulness of high urine output as a low-cost early predictor for postoperative hypoparathyroidism.. Method: A study was conducted inside The American British Cowdray Medical Center I.A.P. for a year in adult patients who underwent total thyroidectomy divided in two groups: those with post-surgical hypocalcemia and without post-surgical hypocalcemia using the 24-hour calcium levels. Urinary output of each patient was verified searching for the relation between this measurement and the probability of developing post-surgical hypoparathyroidism. Results: $A$ total of 47 patients were studied, of whom 19 (40\%) were classified with post-surgical hypocalcemia and 28 (59.5\%) with no post-surgical hypocalcemia. The urinary output mean on the first 8 hours post-operatory was higher in the post-surgical hypocalcemia group in comparison with the group with no evidence of hypocalcemia, showing no significant difference $(p=0.392)$. Urinary output 16 hours post-surgical was no significant either $(p=0.435)$. Conclusions: In our study, there was no relation found between the increase of

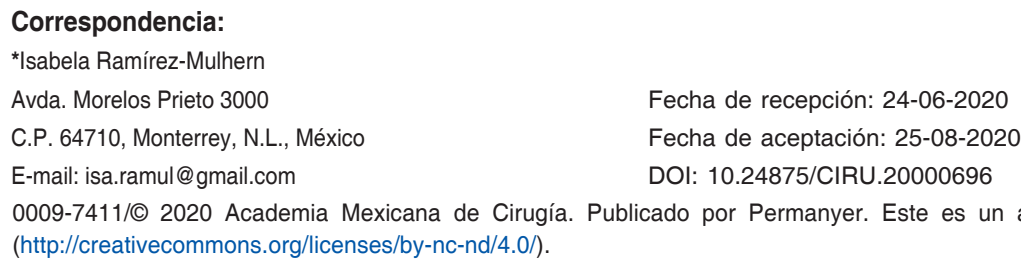


the urinary output and the post-surgical hypoparathyroidism prediction. Further studies with a bigger sample and a stronger methodologic design (prospective) are needed to determine if the difference obtained may be useful as a predictor.

Key words: Hypocalcemia. Hypoparathyroidism. Parathormone. Thyroidectomy.

\section{Introducción}

La cirugía de tiroides representa una de las cirugías electivas más frecuentes alrededor del mundo; tan solo en Alemania se realizan cerca de 60,000 tiroidectomías anuales'. A pesar de que las complicaciones transoperatorias son bajas y la mortalidad es casi nula, el hipoparatiroidismo posoperatorio (PoSH, post-surgical hypoparathyroidism) es la complicación posoperatoria más frecuente de la tiroidectomía total ${ }^{2}$.

Actualmente no existe un consenso internacional para la definición de PoSH, por lo que establecer una incidencia precisa resulta complejo. En una revisión sistemática de 115 estudios se obtuvo una incidencia de PoSH temporal del $27 \%^{3}$, y del $23.6 \%$ en el año 2017 según la British Association of Endocrine and Thyroid Surgeons (BAETS), que propone la definición de PoSH como un valor de calcio sérico $<8.4 \mathrm{mg} / \mathrm{dl}$ al primer día de posoperatorio ${ }^{4}$, la cual será utilizada para fines de este estudio.

En el año 2010, la Thyroid Cancer Alliance reportó un seguimiento a 1,995 pacientes posoperados de tiroidectomía, de los cuales hasta el 39\% cursaron con PoSH transitorio, con una progresión a permanente en el $14 \% 5$.

El origen del PoSH es principalmente una lesión directa de las glándulas paratiroides o de su vascularización durante la tiroidectomía, lo cual condiciona hipoxia de una o más glándulas paratiroideas, fenómeno conocido como aturdimiento o stunning, provocando que se produzca una disminución en las concentraciones séricas de hormona paratiroidea (PTH), calcio, fósforo y magnesio ${ }^{6}$.

Es bien sabido que la hipocalcemia suele tener un retraso de hasta 24 horas en comparación con el descenso de la PTH, por la fisiología del calcio y su unión a las proteínas, y por lo tanto se han propuesto estrategias predictivas para el reconocimiento temprano (determinación de PTH intacta sérica, indicando que un descenso por debajo de $10 \mathrm{pg} / \mathrm{ml}$ a las 4 horas de posoperatorio predice positivamente la aparición de PoSH con una sensibilidad del 94\% y una especificidad del $100 \%)^{7}$.
El objetivo de este estudio es la búsqueda de predictores tempranos de bajo costo siguiendo el principio fisiológico de la acción renal de la PTH para inducir una mayor expresión de los receptores transitorios de calcio de la superficie celular subfamilia V, miembros 5 y 6 (TRPV5 y TRPV6) altamente sensibles al calcio ${ }^{8}$, permitiendo una entrada pasiva a la célula por gradiente. El calcio debe pasar al líquido peritubular a través de la membrana basolateral por el antitransportador $\mathrm{Na}^{+} / \mathrm{Ca}^{++}$(NCX1) y la ATPasa de $\mathrm{Ca}^{++}$(PMCA), las cuales a su vez se activan por acción indirecta de la PTH ${ }^{9}$.

La ausencia del estímulo hormonal previamente mencionado en el contexto de PoSH condiciona una disminución de captación de calcio en el túbulo distal, dejando de ocurrir el intercambio $\mathrm{Na}^{+} / \mathrm{Ca}^{++}$en la membrana basolateral de la célula y ocasionando una baja concentración intracelular de sodio, por lo tanto, una menor fuerza oncótica intracelular en el túbulo distal $y$, finalmente, una menor absorción de agua y un incremento en el gasto urinario.

\section{Método}

\section{Diseño}

Se realizó un estudio retrospectivo unicéntrico en The American British Cowdray Medical Center I.A.P., a 1 año, en adultos de 18-99 años, tanto hombres como mujeres, que hubieran sido sometidos a tiroidectomía total entre el 1 de enero de 2018 y el 31 de diciembre de 2018.

\section{Criterios de inclusión}

Pacientes sometidos a tiroidectomía total con o sin disección del compartimento central o de los compartimentos laterales.

\section{Criterios de exclusión}

- Pacientes con albúmina sérica anormal (rango normal: $3.4-4.8 \mathrm{~g} / \mathrm{dl})$. 
- Pacientes con calcio sérico preoperatorio anormal (rango normal: 8.8-10.2 mg/dl).

- Pacientes con valores preoperatorios anormales de PTH (rango normal: 9.5-75 pg/ml).

- Pacientes con insuficiencia renal.

- Pacientes sin toma de calcio posoperatorio.

- Pacientes sin cuantificación de la diuresis en el posoperatorio inmediato.

Se verificaron los registros de la diuresis del posoperatorio inmediato de las primeras 8,16 y 24 horas, y se calcularon los gastos urinarios de cada paciente, utilizando los valores de calcio sérico corregido por albúmina de cada paciente para poder realizar una tabla de correlación. Se identificaron los pacientes que recibieron calcio suplementario en las primeras horas de posoperatorio.

\section{Análisis estadístico}

Los pacientes se dividieron en dos grupos: los que presentaron PoSH y los que cursaron con valores normales de calcio en el primer día de posoperatorio. Se analizó el gasto urinario de las primeras 8 horas en ambos grupos y se determinó el hipoparatiroidismo con los parámetros de la BAETS (calcio sérico $<8.4 \mathrm{mg} / \mathrm{dl}$ en el día 1 de posoperatorio).

Igualmente, se hizo distinción entre los pacientes que recibieron suplementación oral de calcio profiláctica.

Se utilizó la prueba de Kolmogórov-Smirnov para corroborar la normalidad de la variable del gasto urinario; sin embargo, se identificó una distribución no normal en el grupo de hipocalcemia posoperatoria y una distribución normal limítrofe en el grupo sin hipocalcemia posoperatoria, por lo que se utilizó la prueba $\mathrm{U}$ de Mann-Whitney para dos variables independientes utilizando el software IBM SPSS Statistics Subscription 25.

La razón de momios se presenta con un intervalo de confianza del $95 \%$, y se consideró significativo un valor $p<0.05$.

\section{Resultados}

Se recabaron expedientes de 73 pacientes que se sometieron a tiroidectomía total durante el año 2018 en The American British Cowdray Medical Center I.A.P., de los cuales se obtuvieron 47 que cumplieran con los criterios de inclusión: el $40 \%$ integraron el grupo con hipocalcemia posoperatoria y el $59.5 \%$ el grupo sin hipocalcemia posoperatoria.
En ambos grupos, el sexo predominante fue el femenino: $79 \%$ en el grupo de hipocalcemia posoperatoria y $78 \%$ en el grupo sin hipocalcemia posoperatoria.

La media de edad en el grupo de pacientes con hipocalcemia posoperatoria fue menor (45 años) que la del grupo sin hipocalcemia (50 años), sin presentar diferencia significativa $(p=0.332)$ para ser tomada como factor de riesgo. Del mismo modo, el grupo etario con mayor prevalencia de hipocalcemia posoperatoria fue el de 41-60 años (41\%).

La media de gasto urinario en las primeras 8 horas de posoperatorio fue mayor en el grupo de hipocalcemia posoperatoria $(1.61 \pm 0.25 \mathrm{ml} / \mathrm{kg} / \mathrm{h})$ que en el grupo sin hipocalcemia $(1.21 \pm 0.13 \mathrm{ml} / \mathrm{kg} / \mathrm{h})($ Fig. 1); sin embargo, no fue una diferencia significativa $(p=0.392)$. En el gasto urinario de las primeras 16 horas tampoco se identificó una diferencia significativa ( $p=0.435)$.

Del mismo modo, el gasto urinario de 24 horas fue mayor en el grupo de hipocalcemia posoperatoria $(2.07 \pm 0.32 \mathrm{ml} / \mathrm{kg} / \mathrm{h})$ que en el grupo sin hipocalcemia $(1.87 \pm 0.20 \mathrm{ml} / \mathrm{kg} / \mathrm{h})$, pero de nuevo la diferencia no fue significativa ( $p=0.501$ ) (Fig. 2).

Entre ambos grupos, el $36.1 \%$ (17) de los pacientes recibieron suplementación con calcio dentro de las primeras horas de posoperatorio, de los cuales el $41 \%$ cursó con hipocalcemia posoperatoria a pesar de ello, mientras que en el grupo sin hipocalcemia posoperatoria el $64.2 \%$ (18) de los pacientes no recibieron suplementación de calcio. Por lo tanto, no se logró obtener una correlación positiva entre la suplementación de calcio y la prevención de la hipocalcemia posoperatoria ( $p=0.938)$.

La media de estancia hospitalaria fue mayor en el grupo con hipocalcemia posoperatoria, con 2.68 días de promedio, frente a 1.82 días en el grupo sin hipocalcemia posoperatoria, siendo esta diferencia significativa $(p=0.020)$.

\section{Discusión}

Actualmente no existe un consenso aceptado internacionalmente acerca de la definición de PoSH. La variabilidad en definiciones es tal que Mehanna, et al. ${ }^{10}$ encontraron unas tasas de incidencia del $0 \%$ al $46 \%$ en una misma cohorte de pacientes dependiendo de la definición utilizada.

Lorente-Poch, et al. ${ }^{11}$ adoptaron el término "falla paratiroidea" para incluir tres síndromes; para fines de este estudio, se utilizó el primero como parámetro: la hipocalcemia posoperatoria (calcio sérico 


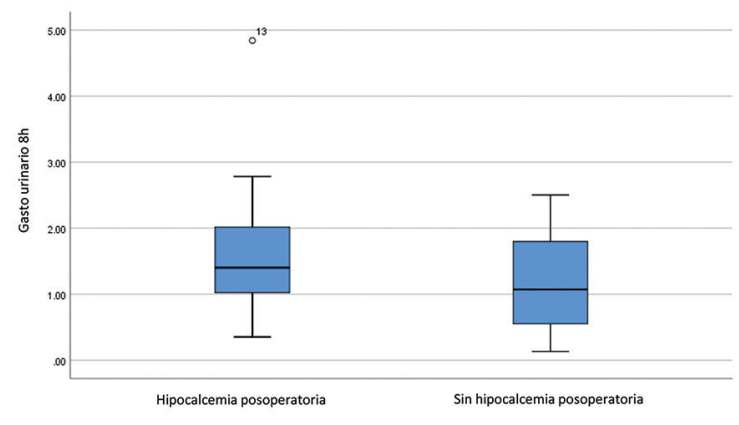

Figura 1. Comparación del gasto urinario a las 8 horas de la tiroidectomía total en pacientes con y sin hipocalcemia posoperatoria $(p=0.392)$.

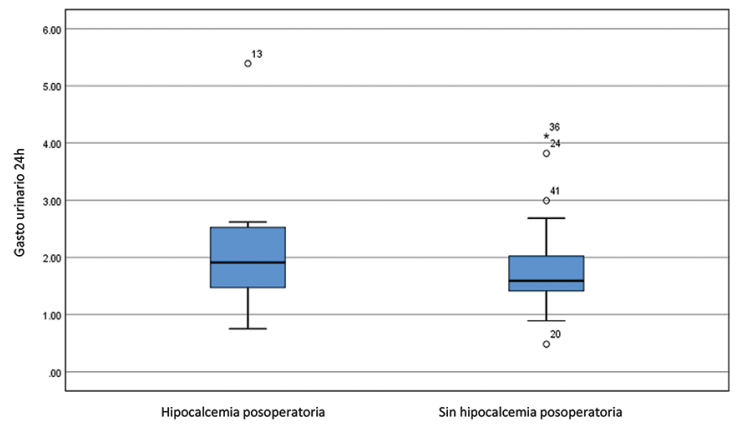

Figura 2. Comparación del gasto urinario a las 24 horas de la tiroidectomía total en pacientes con y sin hipocalcemia posoperatoria $(p=0.501)$.

$<8 \mathrm{mg} / \mathrm{dl}$ dentro de las primeras 24 horas posquirúrgicas, requiriendo suplementación de calcio al momento del alta hospitalaria).

La importancia de este fenómeno radica en la importante incidencia, la morbilidad que conlleva, tomando como ejemplo los altos costos implicados: la estancia intrahospitalaria de nuestro estudio superó las 24 horas $(p=0.20)$. En un estudio se realizó una encuesta a 374 pacientes con PoSH permanente, de los cuales $75 \%$ cursan actualmente con sintomatología a pesar de recibir tratamiento adecuado, $80 \%$ había acudido al servicio de urgencias o sido hospitalizado al menos una vez a consecuencia del PoSH, por lo que notamos la importante inversión que se continúa realizando a largo plazo a consecuencia de la patología ${ }^{11}$.

Mokrysheva, et al. ${ }^{12}$ dieron seguimiento a 76 pacientes con PoSH permanente durante 2 años (2017-2019) y encontraron que las complicaciones más frecuentes fueron nefrolitiasis en el $22 \%$, disminución de la tasa de filtrado glomerular por debajo de $60 \mathrm{ml} / \mathrm{min} / 1.73$ $\mathrm{m}^{2}$ en el $11 \%$, arritmias y prolongación del QT corregido en 5 pacientes (6.5\%), así como síndrome de Fahr (6.5\%) caracterizado por calcificaciones en el sistema nervioso central.

Entre los intentos de predecir este fenómeno, Barczyński, et al. ${ }^{13}$ reportaron que el descenso en la PTH intacta al momento del cierre de la piel y a las 4 horas $(<10 \mathrm{pg} / \mathrm{ml})$ de posoperatorio se correlaciona de manera significativa con la aparición de hipocalcemia posoperatoria a las 24 horas, con un valor predictivo positivo del $90 \%$. Posteriormente este hecho fue corroborado por distintos autores ${ }^{14,15}$. Sieniawski et al., ${ }^{16}$ por su parte, en un estudio prospectivo de 142 pacientes sometidos a tiroidectomía total por enfermedad benigna, concluyeron que el factor con un valor predictivo positivo más alto era un descenso de la PTH a las 6 horas de más del $65 \%$ del valor basal $(p=0.001)$.

En el año 2014, en un análisis sistemático se observó que la ausencia de descenso de la concentración de calcio en tomas seriadas en 24 horas tiene un valor predictivo pobre para predecir la hipocalcemia transitoria ${ }^{17}$.

Por su parte, nuestro estudio buscó identificar el incremento del gasto urinario como predictor de PoSH. Se encontró que el gasto urinario del grupo con PoSH fue mayor en todas las mediciones $(6,8 \mathrm{y}$ 24 horas), pero no se halló una diferencia significativa en ninguna de las mediciones.

Se considera que un factor importante es la técnica quirúrgica, ya que parece influir en la probabilidad de presentar PoSH. En el año 2017, Chen, et al. ${ }^{18}$ publicaron un estudio en el que analizaron la incidencia acumulativa de PoSH a 9 años, evaluando seis procedimientos distintos de tiroidectomía en 9315 pacientes y demostrando que la tiroidectomía subtotal bilateral cursó con la mayor incidencia de $\mathrm{PoSH}$ (13.5\%), mientras que la tiroidectomía subtotal unilateral presentó la menor incidencia (1.2\%), lo que indica que la agresividad del procedimiento se correlaciona con la aparición de PoSH.

En el contexto del hipoparatiroidismo prolongado se ha visto que los factores predictores que se asocian con mayor precisión son la dosis de calcio o de calcitriol recibida al momento del alta hospitalaria y la presencia de valores altos de calcio sérico con valores bajos detectables de PTH intacta al mes de posoperatorio ${ }^{19}$.

Del mismo modo, no se logró hallar una correlación significativa entre la edad y el desarrollo de PoSH, tal como ha sido reportado por otros autore ${ }^{20}$; sin embargo, se apreció que el grupo de edad con mayor 
incidencia de PoSH en nuestro estudio fue el de 41-60 años.

Tal como se ha descrito en la literatura internacional, en nuestra muestra el sexo femenino fue predominante, pero debido a la cantidad de casos de sexo masculino existe un sesgo para determinar si el sexo del paciente pudiera correlacionarse significativamente con la tasa de hipocalcemia posoperatoria en nuestro estudio.

Se han estudiado anteriormente los efectos de la utilización de calcio, vitamina D y PTH en el manejo del PoSH. Edafe, et al. ${ }^{21}$ realizaron una revisión de 1751 publicaciones para examinar los efectos de la administración de estos elementos; sin embargo, se concluyó que no existe evidencia de alta calidad para el manejo de hipoparatiroidismo a corto y largo plazo posterior a una tiroidectomía total, por lo que no se pudo llevar a cabo un análisis, y los autores concluyeron que no existe evidencia de alta calidad para la prevención del PoSH con estas medidas. En nuestro estudio, la suplementación con vitamina $D$ y calcio en el posoperatorio inmediato no reflejó una diferencia significativa en la incidencia de hipocalcemia posoperatoria.

\section{Conclusiones}

La falla paratiroidea representa un verdadero desafío para el cirujano. A pesar de los distintos predictores que se han propuesto para el manejo oportuno de la hipocalcemia posoperatoria, se debe hacer énfasis en llevar a cabo una técnica quirúrgica de manera sistemática, haciendo hincapié en la disección subcapsular y la identificación de la arteria tiroidea inferior, con el fin de mejorar en la preservación in situ de las glándulas paratiroides durante la tiroidectomía total.

El incremento del gasto urinario en nuestro estudio no se correlacionó significativamente con el grupo de hipocalcemia posoperatoria, a pesar de haberse documentado que, en efecto, el gasto urinario del grupo con hipocalcemia posoperatoria fue mayor. Hace falta realizar estudios con una muestra de mayor tamaño y con un diseño metodológico más fuerte para determinar si en realidad esta diferencia puede fungir como predictor.

\section{Conflicto de intereses}

Ningún autor declara tener conflicto de intereses.

\section{Financiamiento}

No existió ningún tipo de financiamiento para este estudio.

\section{Responsabilidades éticas}

Protección de personas y animales. Los autores declaran que para esta investigación no se han realizado experimentos en seres humanos ni en animales.

Confidencialidad de los datos. Los autores declaran que han seguido los protocolos de su centro de trabajo sobre la publicación de datos de pacientes.

Derecho a la privacidad y consentimiento informado. Los autores han obtenido el consentimiento informado de los pacientes y/o sujetos referidos en el artículo. Este documento obra en poder del autor de correspondencia.

\section{Bibliografía}

1. Fortuny JV, Guigard S, Karenovics W, Triponez F. Surgery of the thyroid: recent developments and perspective. Swiss Med Wkly. 2015;145:w14144.

2. Tredici P, Grosso E, Gibelli B, Massaro MA, Arrigoni C, Tradati N. Identification of patients at high risk for hypocalcemia after total thyroidectomy. Acta Otorhinolaryngol Ital. 2011;31:144-8.

3. El Malki HO, Abouqal R. Systematic review and meta-analysis of predictors of post-thyroidectomy hypocalcaemia (Br J Surg 2014;101:307-320). Br J Surg. 2014;101:883.

4. Joint Meeting of the German Association of Endocrine Surgeons (CAEK) and the British Association of Endocrine and Thyroid Surgeons (BAETS). Langenbeck's Arch Surg. 2016;401:1045-91.

5. Banach R, Bartès B, Farnell K, Rimmele H, Shey J, Singer S, et al. Results of the Thyroid Cancer Alliance international patient/survivor survey: psychosocial/informational support needs, treatment side effects and international differences in care. Hormones (Athens). 2013;12:428-38.

6. Leiker AJ, Yen TWF, Eastwood DC, Doffek KM, Szabo A, Evans DB et al. Factors that influence parathyroid hormone half-life: determining if new intraoperative criteria are needed. JAMA Surg. 2013;148:602-6.

7. Lombardi CP, Raffaelli M, Princi P, Santini S, Boscherini M, De Crea C, et al. Early prediction of postthyroidectomy hypocalcemia by one single iPTH measurement. Surgery. 2004;136:1236-41.

8. Lofrese JJ, Basit H, Lappin SL. Physiology, parathyroid. StatPearls. Treasure Island (FL): StatPearls Publishing; 2019. Disponible en: https:// www.ncbi.nlm.nih.gov/books/NBK482510/\#: :text=The\%20parathyroid $\% 20$ glands' $\% 20$ function $\% 20$ is, bone $\% 20$ breakdown $\% 20$ and $\% 20$ calcium\%20release.

9. Magyar CE, White KE, Rojas R, Apodaca G, Friedman PA. Plasma membrane Ca2+-ATPase and NCX1 $\mathrm{Na}+/ \mathrm{Ca} 2+$ exchanger expression in distal convoluted tubule cells. Am J Physiol Renal Physiol. 2002;283:F29-40.

10. Mehanna HM, Jain A, Randeva H, Watkinson J, Shaha A. Postoperative hypocalcemia - The difference a definition makes. Head Neck. 2010;32:279-83

11. Lorente-Poch L, Sancho JJ, Muñoz-Nova JL, Sánchez-Velázquez P, Sitges-Serra A. Defining the syndromes of parathyroid failure after total thyroidectomy. Gland Surg. 2015:4:82-90.

12. Mokrysheva N, Eremkina A, Kovaleva E. The long-term outcomes of chronic post-surgical hypoparathyroidism. Endocrine Abstracts. 2019. Disponible en: http://dx.doi.org/10.1530/endoabs.63.gp21

13. Barczyński M, Cichoń S, Konturek A. Which criterion of intraoperative iPTH assay is the most accurate in prediction of true serum calcium levels after thyroid surgery? Langenbecks Arch Surg. 2007;392:693-8.

14. Cannizzaro MA, Okatyeva V, Lo Bianco S, Caruso V, Buffone A. Hypocalcemia after thyroidectomy: IPTH levels and iPTH decline are predictive? Retrospective cohort study. Ann Med Surg. 2018;30:42-5.

15. Carr AA, Yen TW, Fareau GG, Cayo AK, Misustin SM, Evans DB, et al. A single parathyroid hormone level obtained 4 hours after total thyroidectomy predicts the need for postoperative calcium supplementation. J Am Coll Surg. 2014;219:757-64. 
Cirugía y Cirujanos. 2021;89(4)

16. Sieniawski K, Kaczka K, Paduszyńska K, Fendler W, Tomasik B, Pomorski L. Early Predictors of Post. Thyroidectomy Hypoparathyroidism. Pol Przegl Chir. 2016;88(6):305-14.

17. Edafe O, Antakia R, Laskar N, Uttley L, Balasubramanian SP. Systematic review and meta-analysis of predictors of post-thyroidectomy hypocalcaemia. Br J Surg. 2014;101:307-20.

18. Chen K-C, Iqbal U, Nguyen P-A, Hsu C-H, Huang C-L, Hsu Y-HE, et al. The impact of different surgical procedures on hypoparathyroidism afte thyroidectomy: a population-based study. Medicine. 2017;96:e8245.
19. Sitges-Serra A, Ruiz S, Girvent M, Manjón H, Dueñas JP, Sancho JJ. Outcome of protracted hypoparathyroidism after total thyroidectomy. $\mathrm{Br}$ J Surg. 2010;97:1687-95.

20. Coimbra C, Monteiro F, Oliveira P, Ribeiro L, de Almeida MG, Condé A. Hypoparathyroidism following thyroidectomy: predictive factors. Acta Otorrinolaringol Esp. 2017;68:106-11.

21. Edafe O, Mech CE, Balasubramanian SP. Calcium, vitamin D or recombinant parathyroid hormone for managing post-thyroidectomy hypoparathyroidism. Cochrane Database Syst Rev. 2019;(5):CD012845. 